Research

\title{
Silencing long intergenic non-protein coding RNA 00987 inhibits proliferation, migration, and invasion of osteosarcoma cells by sponging miR-376a-5p to regulate FNBP1 expression
}

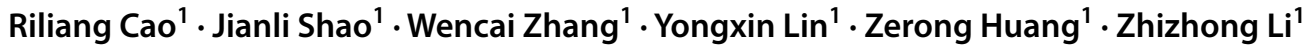

Received: 18 April 2021 / Accepted: 24 May 2021

Published online: 01 June 2021

(C) The Author(s) 2021 OPEN

\begin{abstract}
High expression of long intergenic non-protein coding RNA 987 (LINC00987) is strongly associated with low overall survival of osteosarcoma; however, its role in osteosarcoma remains unclear. This study explored the biological function and underlying mechanism of LINC00987 in osteosarcoma. In this study, LINC00987 expression in osteosarcoma cells was analyzed using Cancer Cell Line Encyclopedia and qRT-PCR. The proliferation and migration and invasion in osteosarcoma cells were evaluated using Cell Counting Kit-8 and Transwell assays, respectively. Bioinformatic analysis was used to predict the LINC00987-bound miRNAs and miR-376a-5p-bound mRNAs. Dual-luciferase reporter assays were used to assess the interaction between miR-376a-5p, LINC00987, and forming-binding protein 1 (FNBP1). FNBP1 expression was measured by western blotting. LINC00987 was found to be upregulated in osteosarcoma cells. LINC00987 silencing suppressed proliferation, migration, and invasion of osteosarcoma cells. Additionally, miR-376a-5p expression was downregulated in osteosarcoma cells. miR-376a-5p knockdown reversed the effect of LINC00987 silencing on the biological function of osteosarcoma cell. miR-376a-5p was found to target LINC00987 and FNBP1. FNBP1 protein level was increased in osteosarcoma cells; however, it was inhibited by silencing LINC00987 and enhanced by silencing miR-376a-5p. In conclusions, this study suggests LINC00987 silencing inhibits osteosarcoma cell proliferation, migration, and invasion by sponging miR-376a-5p to regulate FNBP1 expression. LINC00987 as a potential therapeutic target for osteosarcoma.
\end{abstract}

Keywords FNBP1 $\cdot$ IncRNA $\cdot$ miRNA $\cdot$ Osteosarcoma

\author{
Abbreviations \\ LINC00987 Long intergenic non-protein coding RNA 987 \\ 987FNBP1 Forming-binding protein 1 \\ IncRNA Long noncoding RNA \\ NC Negative control \\ FBS Fetal bovine serum \\ CCLE Cancer Cell Line Encyclopedia \\ GEO Gene Expression Omnibus
}

Supplementary Information The online version contains supplementary material available at https://doi.org/10.1007/s12672-02100412-x.

Zhizhong Li, zhizhongli2014@126.com | 'Department of Orthopedics, First Affiliated Hospital of Jinan University, 613 W. Huangpu Avenue, Tianhe District, Guangzhou 510630, China.

Discover Oncology $\quad$ (2021) $12: 18 \quad$ https://doi.org/10.1007/s12672-021-00412-x 
3'- UTR 3'-Untranslated region

WT Wild-type

\section{Introduction}

Osteosarcoma is a common malignant bone tumor with lung metastasis, high mortality, and poor prognosis and mainly occurs in children and adolescents under 20 years of age [1,2]. To date, surgery combined with neoadjuvant chemotherapy has been the major treatment for osteosarcoma [3]. However, the surgical plan is established; therefore, drug-assisted treatment is the key to improving the survival rate. Hence, further study of the complex pathogenesis of osteosarcoma will help develop novel drugs.

Long noncoding RNAs (IncRNAs) can regulate tumorigenesis, metastasis, and gene expression in various cancer tissues or cells [4,5]. Abnormal expression of IncRNAs involved in tumorigenesis, progression, metastasis, and chemotherapy drug resistance can be used as therapeutic targets and diagnostic and prognostic markers in osteosarcoma [6-8]. A previous study found that high long intergenic non-protein coding RNA 987 (LINC00987) expression strongly correlated with low survival of osteosarcoma [9]. However, their biological functions in osteosarcoma remain unclear.

In this study, the expression of LINC00987 in osteosarcoma cells was analyzed. Next, we focused on further investigating the role and regulatory mechanisms of LINC00987 to provide new references for treating osteosarcoma.

\section{Materials and methods}

\subsection{Cell culture}

The conditionally immortalized human fetal osteoblastic cell line hFOB1.19 (normal cell) and human osteosarcoma cell lines (143B, U2OS, MG63, and Saos-2) were purchased from the Institute of Cell Bank/Institutes for Biological Sciences (Shanghai, China). Cells were cultured as described in our previous study [10].

\section{2 qRT-PCR assay}

Total RNA was extracted from hFOB1.19, 143B, MG63, Saos-2, and U2OS cells using TRIzol reagent (Invitrogen, Carlsbad, (A, USA) and dissolved in $20 \mu \mathrm{L} \mathrm{DEPC} \mathrm{H}_{2} \mathrm{O}$. The first-strand cDNA synthesis reaction system was configured in an RNasefree PCR tube, and mRNA was reverse-transcribed to CDNA using the EasyScript First-Strand cDNA Synthesis SuperMix kit (Transgen, Beijing, China). IncRNA, miRNA, and mRNA expression was detected using SYBR Green qPCR SuperMix (Invitrogen) with the ABI PRISM 7500 Sequence Detection System (Applied Biosystems, Foster City, CA, USA). $\beta$-actin was used as an endogenous control for IncRNA and mRNA, while U6 was used as a miRNA. All experiments were performed in triplicate. The relative expression was calculated using the $2^{-\Delta \Delta C t}$ method [11]. PCR primer sequences were purchased from Sangon (Shanghai, China) and are listed in Table 1. Each experiment was repeated three times.

\subsection{Transfection}

Three LINC00987 siRNAs (si-LINC00987-1, -2, and -3) and a negative control siRNA (si-NC) were purchased from Shanghai Genepharma (Shanghai, China) and are shown in Table 1 (Shanghai, China). Thereafter, 50 ng each of si-NC, siLINC00987-1, si-LINC00987-2, and si-LINC00987-3 was transfected into 143B and U2OS cells, respectively. After transfection for 48 h, LINC00987 expression was measured by qRT-PCR. Moreover, miR-376a-5p inhibitor (miR inhibitor) and negative control inhibitor (NC inhibitor) were purchased from Shanghai GenePharma (Shanghai, China). Thereafter, 50 ng each of si-LINC00987-3 and NC inhibitor (si-LINC00987-3 + NC inhibitor) or miR inhibitor (si-LINC00987-3 + miR inhibitor) was co-transfected into 143B or U2OS cells, respectively. After transfection for $48 \mathrm{~h}$, miR-376a-5p expression was measured by qRT-PCR. 
Table 1 Synthesized sequences

\begin{tabular}{lll}
\hline LINC00987 & siRNA sequence & \\
\cline { 2 - 3 } & Sense (5'-3') & Antisense (5'-3') \\
\hline si-LINC00987-1 & GCUCUGUGCAUCAGGUAAATT & UUUACCUGAUGCACAGAGCTT \\
si-LINC00987-2 & GGAUUUAGCCUUGUGCCAATT & UUGGCACAAGGCUAAAUCCTT \\
si-LINC00987-3 & GGUAAUGAAUGGUGACUAUTT & AUAGUCACCAUUCAUUACCTT \\
\hline qRT-PCR & Primer sequences & Reverse primer $\left(5^{\prime}-3^{\prime}\right)$ \\
\cline { 2 - 3 } & Forward primer (5'-3') & TTCCTAGAACTCACCCTTCCT \\
\hline LINC00987 & GTTCTGATTTCCTGGGTTACC & TCTGACACAGTGCGCTTCAT \\
FNBP1 3 -actin & ATTGGGAAGTGCCTGGATGG & TCGTCCCAGTTGGTGACGAT \\
miR-370-3p & GCATGGGTCAGAAGGATTCCT & CTCAACTGGTGTCGTGGA \\
miR-376a-5p & ACACTCCAGCTGGGGCCTGCTGGGGTGGAACC & CTCAACTGGTGTCGTGGA \\
miR-543 & ACACTCCAGCTGGGGTAGATTCTCCTTCTATGA & CTCAACTGGTGTCGTGGA \\
U6 & ACACTCCAGCTGGGAAACATTCGCGGTGCACT & AACGCTTCACGAATTTGCGT \\
\hline
\end{tabular}

\subsection{Cell proliferation, migration, and invasion assays}

For cell proliferation assay, $24 \mathrm{~h}$ after transfection, MG-63 and Saos 2 cells were seeded at $1 \times 10^{3}$ cells per well in 96-well plates. The cell proliferation assay was performed at 24, 48, and $72 \mathrm{~h}$ using the Cell Counting Kit-8 (CCK8; Dojindo, Kumamoto, Japan) at $450 \mathrm{~nm}$. Transwell migration and invasion assays were performed as previously described [10]. The bottom of the upper chamber was pre-coated with or without Matrigel (BD Biosciences, San Diego, CA, USA) for migration and invasion assays. The transfected cells $\left(5 \times 10^{4}\right.$ cells $)$ in $200 \mu \mathrm{L}$ of $0.1 \%$ fetal bovine serum (FBS)-containing medium were placed in the upper chamber. The lower chamber was filled with $10 \%$ FBS (600 $\mu \mathrm{L})$. After $48 \mathrm{~h}$ of culture, cells in the upper chamber were removed. The cells that passed through the membrane filter were fixed and stained and then randomly observed ( $200 \times$ magnification) using a LEICA Microscope (Tokyo, Japan) for each well.

\subsection{Bioinformatic database assay}

The Cancer Cell Line Encyclopedia (CCLE) database (www.portals.broadinstitute.org/ccle) was used to analyze the levels of LINC00987 transcripts in osteosarcoma cells. The miRNA expression in osteosarcoma cells was analyzed by using GEO2R in GSE70367 of Gene Expression Omnibus (GEO, https://www.ncbi.nlm.nih.gov/geo/), and the downregulated expression of miRNAs (fold change $\leq-1$ ) was selected. The potential miRNAs bound to LINC00987 were analyzed using the LncBase Experimental v.2 subset of DIANA Tools [12]. The potential mRNAs bound to miR-376a-5p were analyzed using Targetscan 7.2 [13], miRDB [14], and miRWalk (http://mirwalk.umm.uni-heidelberg.de/), and the intersection was taken for further verification. Additionally, the intersection mRNA expression in osteosarcoma cells was analyzed using GEO2R in GSE70367 and Oncomine (https://www.oncomine.org/).

\subsection{Plasmid construction and luciferase reporter assay}

The 3'-untranslated region (UTR) sequences of wild-type LINC00987 and forming-binding protein 1 (FNBP1) (WTLINC00987 and WT-FNBP1) and mutated LINC00987 and FNBP1 (MUT-LINC00987 and MUT-FNBP1) were synthesized by GENEWIZ (Suzhou, China) and cloned into the luciferase reporter vector psi-CHECK2. For the luciferase reporter assay, 293 T cells were plated at a density of $5 \times 10^{4}$ cells per well in 24 -well plates. The next day, 293 T cells were cotransfected using Lipofectamine 2000 (Invitrogen) with the following combinations: WT-LINC00987 plus NC mimic or miR-376a-5p mimics, MUT-LINC00987 plus NC mimic or miR-376a-5p mimics, WT-FNBP1 plus NC mimic or miR-376a-5p 
mimics, and MUT-FNBP1 plus NC mimic or miR-376a-5p mimics. Forty-eight hours after transfection, luciferase assays were performed using a dual-luciferase reporter assay system (Promega).

\subsection{Western blotting}

Western blotting was performed as described previously [10]. Briefly, total proteins were extracted from osteosarcoma cells. Extracted proteins $(30 \mu \mathrm{g})$ were separated using 10\% SDS-PAGE and transferred onto a polyvinylidene difluoride membrane (Millipore, Billerica, MA, USA). After blocking, membranes were incubated with anti-FNBP1 antibody (Cat\# ab100918, $0.5 \mu \mathrm{g} / \mathrm{mL}$, Abcam, Cambridge, MA, USA) for $1 \mathrm{~h}$ at $37^{\circ} \mathrm{C}$. After washing in TBS with $0.5 \%$ Tween 20, the membrane was incubated with a horseradish peroxidase-conjugated secondary antibody. Finally, chemiluminescence detection was performed using enhanced chemiluminescence, and signals were recorded on X-ray films.

\subsection{Statistical analysis}

Statistical analysis was performed using the SPSS software (version 19.0; IBM, Chicago, IL, USA). Normally distributed data are expressed as the mean \pm standard deviation. The differences between multiple groups (more than two groups) were compared using one-way ANOVA followed by Tukey's post-hoc test. The differences between the two groups were compared using $t$-tests. Statistical significance was set at $\mathrm{P}<0.05$.

\section{Results}

\subsection{LINC00987 expression is increased in osteosarcoma cells}

The qRT-PCR results showed that LINC00987 expression in osteosarcoma cells (143B, U2OS, MG63, and Saos-2) was significantly higher than that in hFOB1.19 cells (normal cell) (Fig. 1A). A previous study found that higher LINC00987 expression was strongly correlated with the lower overall survival of osteosarcoma [9]. In this study, the expression of LINC00987 was consistent with previous studies. Additionally, LINC00987 expression in 143B and U2OS cells was significantly higher than that in in MG63 and Saos-2 cells (Fig. 1A). The results from CCLE-database analysis showed that LINC00987 expression in U2OS cells was obviously higher than that in other osteosarcoma cells (Fig. 1B). Hence, this study selected 143B and $\mathrm{U} 2 \mathrm{OS}$ cells for further investigations.
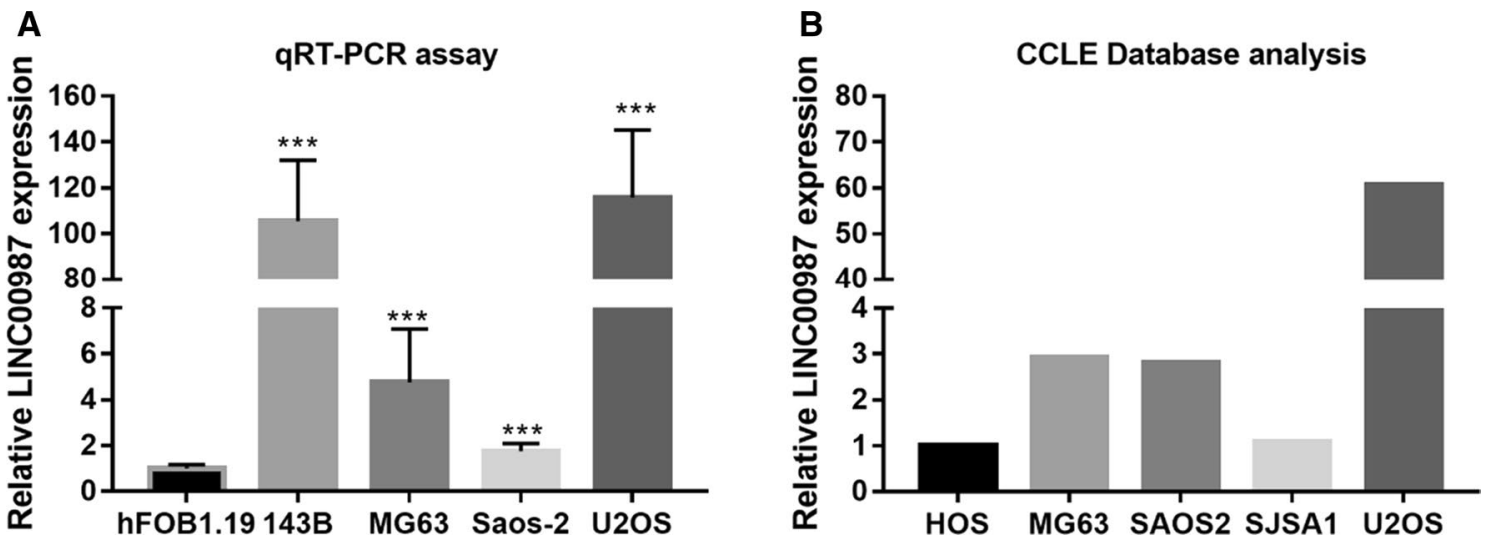

Fig. 1 LINC00987 expression is increased in osteosarcoma cells, especially in 143B and U2OS cells. A LINC00987 expression in the conditionally immortalized human fetal osteoblastic cell line hFOB1.19 (normal cell) and human osteosarcoma cell lines (143B, U2OS, MG63, and Saos-2) measured using qRT-PCR. ${ }^{* * *} \mathrm{P}<0.001$ vs. hFOB1.19 group. B LINC00987 expression in osteosarcoma cell lines were analyzed using the Cancer Cell Line Encyclopedia (CCLE) Database 

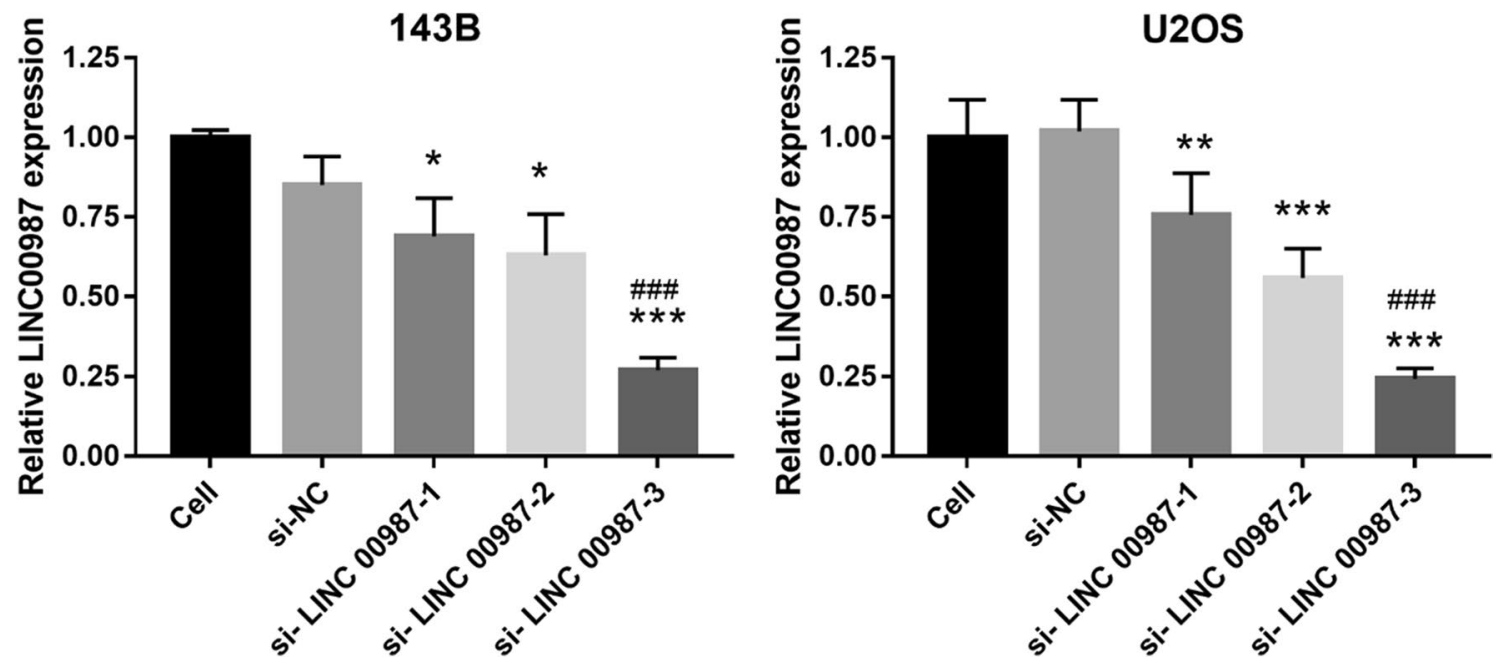

Fig. 2 LINC00987 expression is inhibited by LINC00987 siRNA (si-LINC00987) transfection. LINC00987 expression was measured by qRT-PCR $24 \mathrm{~h}$ after transfection of $143 \mathrm{~B}$ and U2OS cells with three si-LINC00987 sequences and negative control siRNA (si-NC). ${ }^{* * * P<0.001 ~ v s . ~ s i-N C ~}$ group

\subsection{LINC00987 silencing inhibits proliferation, migration, and invasion of $143 B$ and U20S cells}

To study the effect of LINC00987 on the biological function of osteosarcoma cells, LINC00987 expression was knocked down in 143B and U2OS cells. Compared with the cell group, si-NC group showed no significant change in LINC00987 expression, whereas the si-LINC00987 group showed significantly reduced LINC00987 expression (Fig. 2). Compared with the si-LINC00987-1 group, the si-LINC00987-2 group showed no significant change in LINC00987 expression, while the si-LINC00987-3 group showed significantly reduced LINC00987 expression (Fig. 2). Hence, si-LINC00987-3 transfection was selected for further analysis. Additionally, compared with the cell group, the si-NC group showed no significant change in proliferation, migration, and invasion, while the si-LINC00987 group showed significantly reduced proliferation, migration, and invasion, after transfection at 48 and $72 \mathrm{~h}$ (Fig. 3).

\subsection{LINC00987 acts as sponge for miR-376a-5p}

The intersection between the potential miRNAs analyzed by LncBase Experimental v.2 and downregulated miRNAs analyzed by GEO are shown in Fig. 4A. The results showed that miR-370-3p, miR-376a-5p, and miR-543 might bind to LINC00987. Moreover, miR-370-3p, miR-376a-5p, and miR-543 expression was lower in all osteosarcoma cells, except for MG63 cells, than in human mesenchymal stem cells (hMSC), according to the GEO-analyzed results (Fig. 4B). miR-376a-5p and miR-543 expression was significantly reduced in 143B and U2OS cells, and miR-370-3p expression was significantly reduced in U2OS cells, whereas there was no significant change in 143B cells, compared to those in hFOB1.19 cells (Fig. 4C). In addition, the fold reduction in miR-376a-5p expression was lower than that of miR-543 (Fig. 4C). Hence, we chose miR-376a-5p for further studies. The wild-type and mutated binding sites between miR-376a-5p and LINC00987 are shown in Fig. 4D. Co-transfection of miR-376a-5p mimic inhibited the relative luciferase activity of WT-LINC00707transfected cells, whereas it did not affect the activity of MUT-LINC00707-transfected cells (Fig. 4E). Furthermore, miR376a-5p expression in 143B and U2OS cells did not differ between the cell, si-NC, and si-LINC00987-3 groups (Fig. 4F). These results showed that miR-376a-5p expression was reduced in 143B and U2OS cells and that miR-376a-5p can bind to LINC00987.

\section{4 miR-376a-5p reverses the effect of LINC00987 in 143B and U2OS cells}

To study the relationship between LINC00987 and miR-376a-5p, si-LINC00987-3 + NC inhibitor and si-LINC00987-3+ miR inhibitor were co-transfected in $143 \mathrm{~B}$ and U2OS cells, respectively. The results of $\mathrm{QRT}$-PCR showed that 


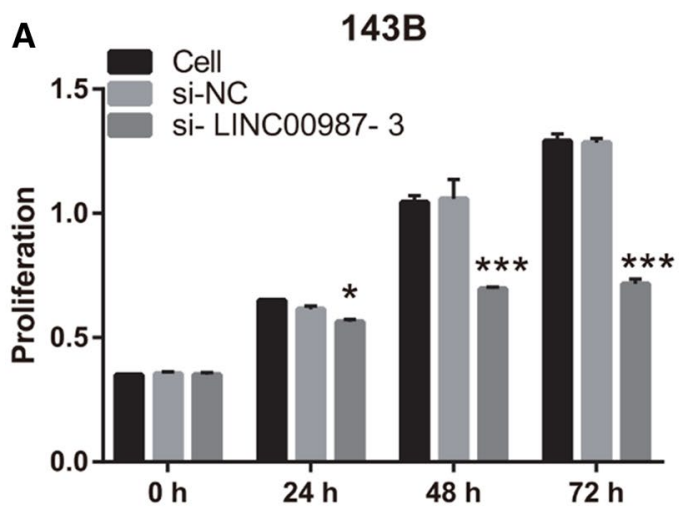

\section{U2OS}
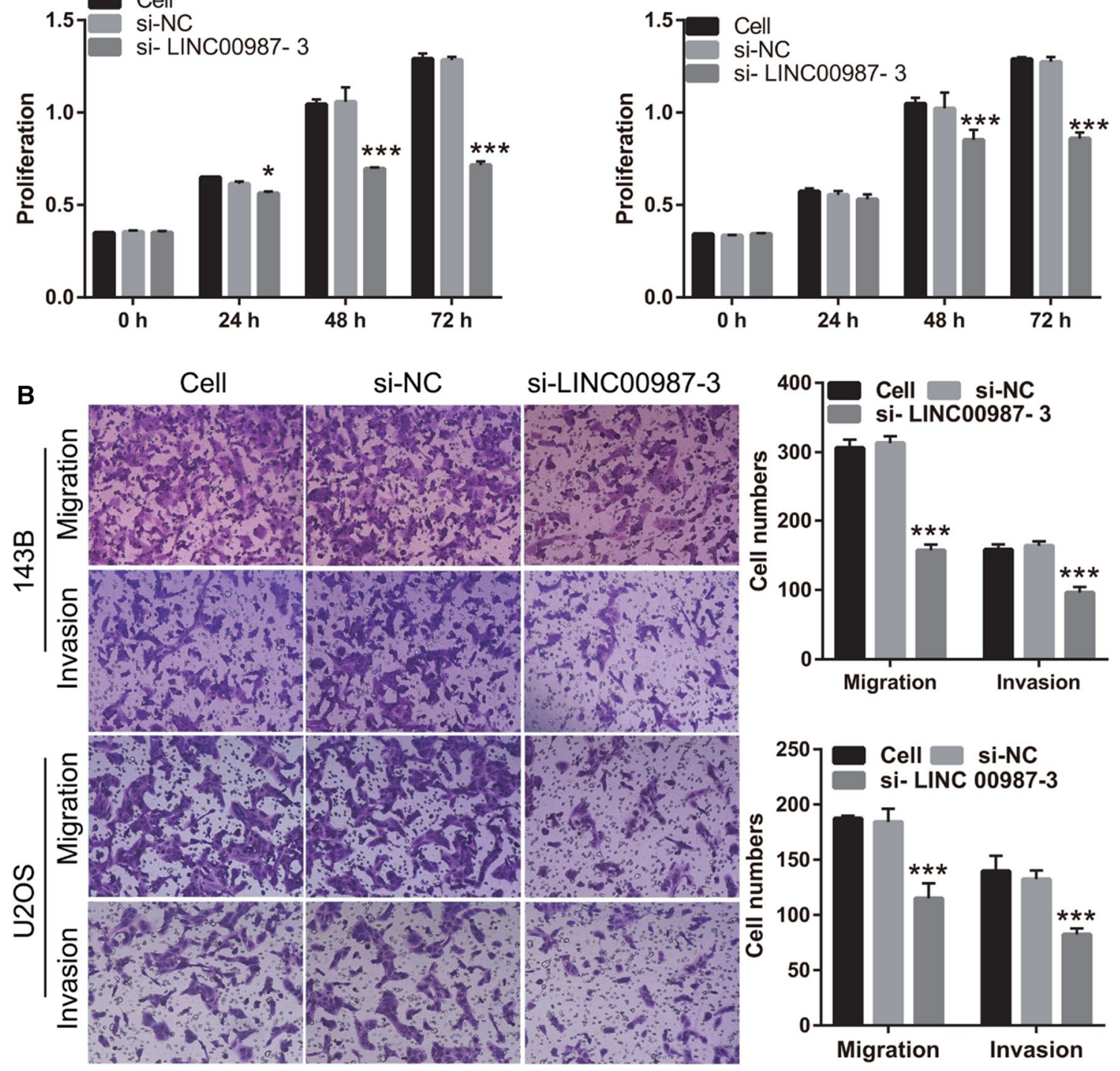

Fig. 3 Silencing of LINC00987 inhibits the proliferation, migration, and invasion of 143B and U2OS cells. A Proliferation measured 24, 48, and $72 \mathrm{~h}$ after transfection, using CCK8. B Migration and invasion measured using Transwell assay $48 \mathrm{~h}$ after transfection. ${ }^{*} \mathrm{P}<0.05,{ }^{* * *} \mathrm{P}<0.001$ vs. si-NC group

miR-376a-5pexpression was knocked down in si-LINC00987-3-transfected 143B and U2OS cells (Fig. 5A). Cell proliferation was significantly increased in the si-LINC00987-3 + miR inhibitor group after transfection at 48 and $72 \mathrm{~h}$ compared to that in the si-LINC00987-3 + NC inhibitor group (Fig. 5B). Furthermore, migration and invasion of the si-LINC00987-3+ miR inhibitor group were significantly increased compared to those in the si-LINC00987-3 + NC inhibitor group $48 \mathrm{~h}$ after transfection (Fig. 5C).

\subsection{FNBP1 is the target of miR-376a-5p}

The intersection between the potential mRNAs analyzed by Targetscan 7.2, miRDB, and miRWalk is shown in Fig. 6A. The results showed that FNBP1 and CCDC88A mRNA expression was higher in osteosarcoma cells than that in human mesenchymal stem cells, according to the GEO-analyzed results (Fig. 6B). The wild-type and mutated binding sites between miR-376a-5p and FNBP1 3'-UTR are shown in Fig. 6C. Co-transfection of miR-376a-5p mimic inhibited the relative luciferase activity of WT-FNBP1 3'-UTR-transfected cells; however, it did not affect MUT-FNBP1 3'-UTR-transfected cells (Fig. 6D). Moreover, the wild-type binding sites between miR-376a-5p and CCDC88A 3'-UTR had only six 
A

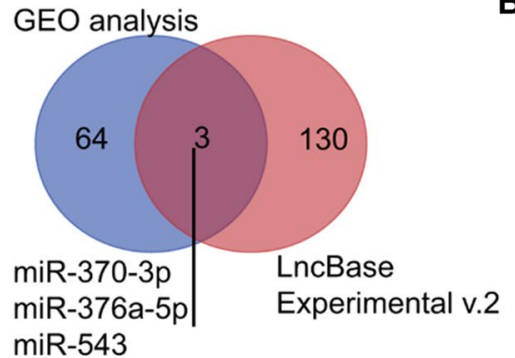

C

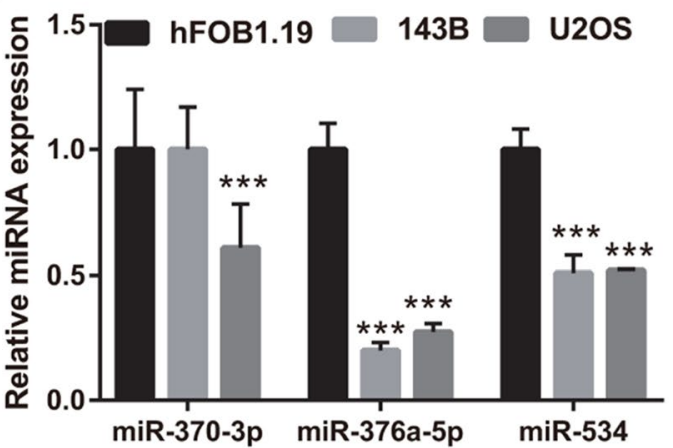

E

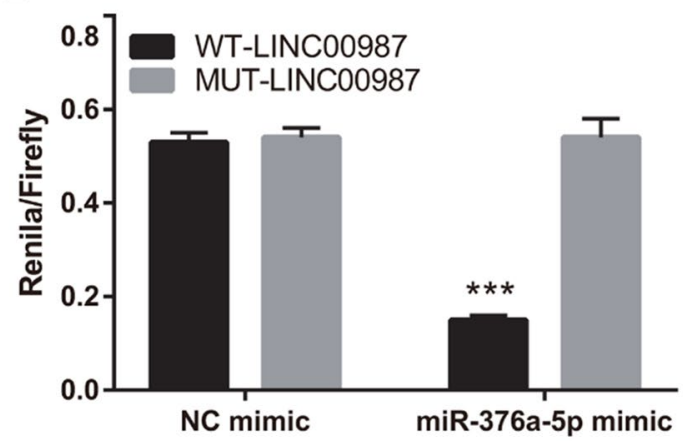

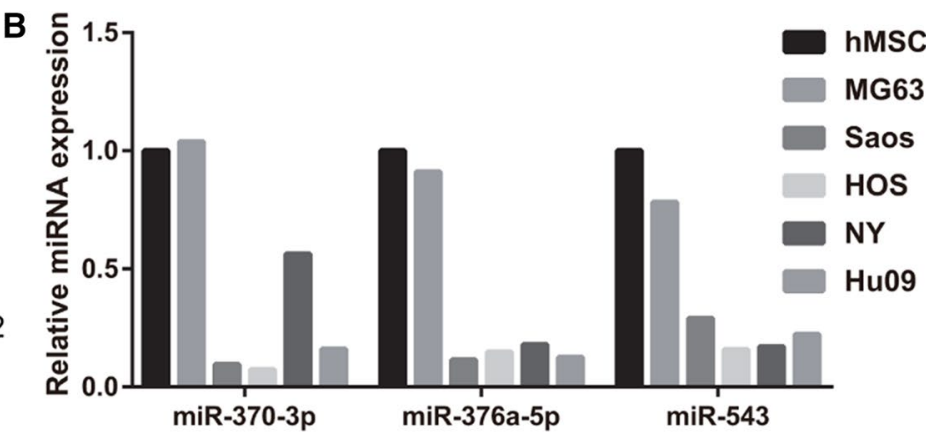

D

\author{
MUT-LINC00987: UUUCUUCAAAUUCUUAGAUG \\ WT-LINC00987: UUUCUUCAAAUAGAAUCUAG \\ || || ||| \\ miR-376a-5p: UGAGUAUCUUCCUCUUAGAUG
}

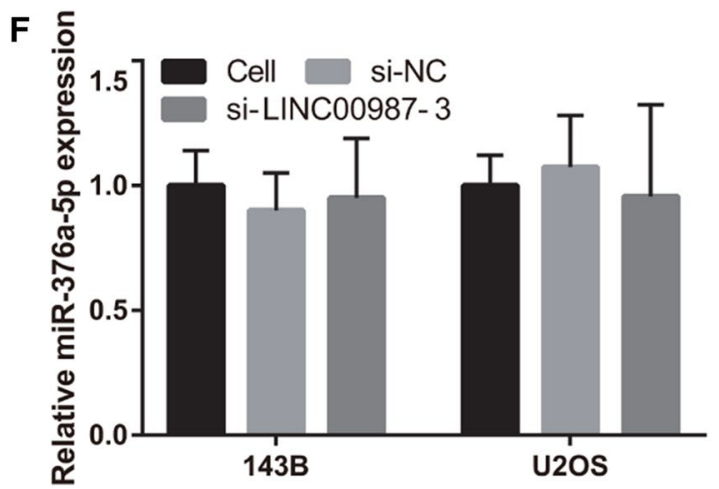

Fig. 4 LINC00987 acts as sponge for miR-376a-5p. A The intersection between the potential miRNAs analyzed using LncBase Experimental v.2 and downregulated-expression miRNAs analyzed using GEO. B miRNA expression in osteosarcoma cells and human mesenchymal stem cells (hMSCs) analyzed using GEO. C miRNA expression in osteosarcoma cells and the conditionally immortalized human fetal osteoblastic cell line hFOB1.19 measured using qRT-PCR. D The wild-type and mutated binding sites between miR-376a-5p and LINC00987. E Luciferase reporter assay results. F miR-376a-5p expression in 143B and U2OS cells measured using qRT-PCR $24 \mathrm{~h}$ after transfection

bases, and there was no significant difference in the relative luciferase activity between cells co-transfected with miR-376a-5p mimic and WT-CCDC88A 3'-UTR and those co-transfected with miR-376a-5p mimic and MUT-CCDC88A 3'-UTR (results not shown). Additionally, compared with normal cell (hFOB1.19 cell), CCDC88A protein levels had no significant change in 143B and MG63 while had significant change in U2OS and Saos-2 (Additional file 1: Fig. S1). These results indicated that miR-376a-5p cannot target CCDC88A 3'-UTR and the expression trends of CCDC88A and LINC00987 in osteosarcoma are not similar. Hence, CCDC88A is not a target gene regulated by LINC00987/miR376a-5p. FNBP1 protein levels were significantly increased in osteosarcoma cells, particularly in $143 B$ and U2OS cells, compared to those in hFOB1.19 cells, which was similar with the expression trends of LINC00987 (Fig. 6E). Compared with the cell group, the si-LINC00987-3 group showed significantly increased FNBP1 protein levels (Fig. 6E). And compared with the si-LINC00987-3 + NC inhibitor group, the si-LINC00987-3 + miR-376a-5p inhibitor group showed significantly decreased FNBP1 protein levels (Fig. 6E). These results suggest that FNBP1 is the target of miR-376a-5p. And LINC00987 and miR-376a-5p can regulate FNBP1 protein levels. 
A

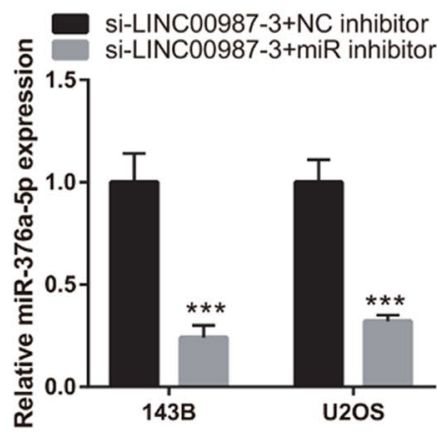

B

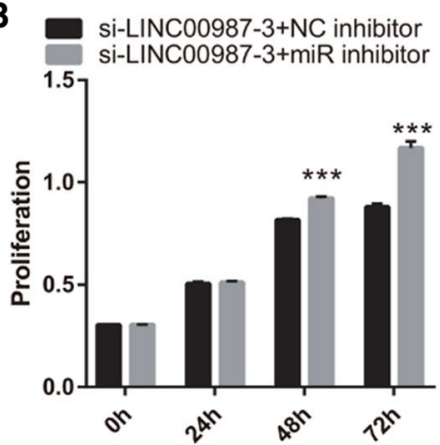

C

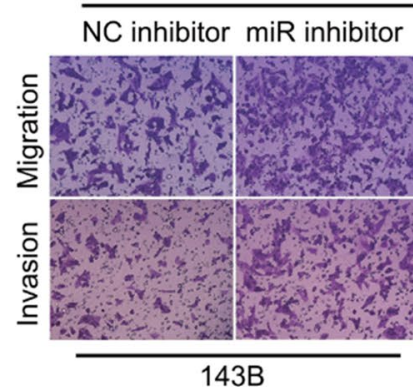

Si-LINC00987-3

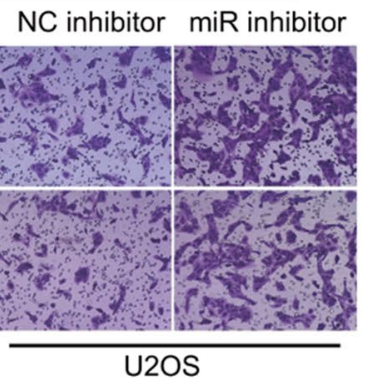

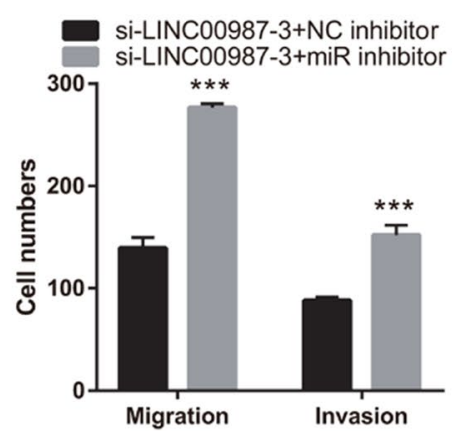

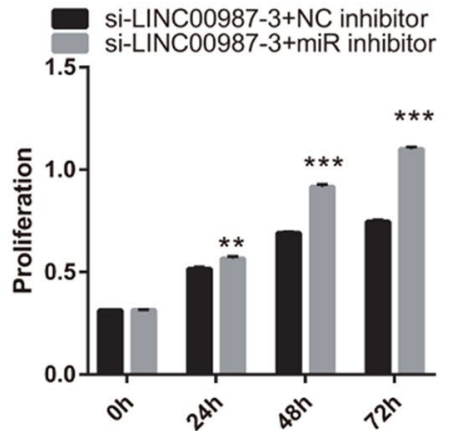

si-LINC00987-3+NC inhibitor si-LINC00987-3+miR inhibitor

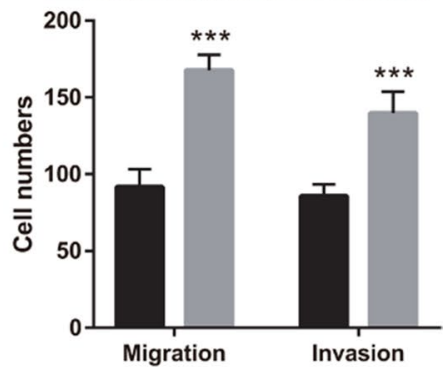

Fig. 5 miR-376a-5p reverses the effect of LINC00987 in 143B and U2OS cells. si-LINC00987-3 and NC inhibitor (si-LINC00987-3 + NC inhibitor) or miR-376a-5p inhibitor (si-LINC00987-3 + miR inhibitor) were co-transfected in 143B and U2OS cells, respectively. A miR-376a-5p expression was measured using qRT-PCR $24 \mathrm{~h}$ after transfection. B Proliferation measured 24,48 , and $72 \mathrm{~h}$ after transfection, using CCK8. C Migration and invasion measured using Transwell assay $48 \mathrm{~h}$ after transfection. ${ }^{* * \mathrm{P}}<0.01,{ }^{* * * P}<0.001$, vs si-LINC00987-3+NC inhibitor

\section{Discussion}

The remission and treatment of osteosarcoma have significantly improved over the last 10 years [2]. However, osteosarcoma does not have a favorable prognosis because of unresolved problems, such as complex pathogenesis and lack of new adjuvant drugs. Therefore, further studies on the mechanism of osteosarcoma development are necessary to develop new therapeutic targets and adjuvant therapeutic drugs. This study focused on the mechanistic involvement of LINC00987 in osteosarcoma. First, it was found that LINC00987 silencing inhibited the proliferation, migration, and invasion of 143B and U2OS cells. Moreover, the inhibition of miR-376a-5p remarkably recovered cell proliferation, migration, and invasion in LINC00987-silenced cells. Additionally, this study found that FNBP1 is the target of miR-376a-5p. FNBP1 expression was increased in osteosarcoma cells; however, it was inhibited by silencing LINC00987 and enhanced by silencing miR$376 a-5 p$. The results revealed an abundance of LINC00987 through sponging miR-376a-5p and positive regulation of FNBP1 protein level, consequently contributing to the proliferation, migration, and invasion of osteosarcoma cells (Fig. 7).

Osteosarcoma overgrowth and metastasis are the most important biological characteristics of osteosarcoma [15]. However, the potential mechanisms underlying these phenomena have not been completely elucidated. The effect of abnormal IncRNA expression in osteosarcoma has received increasing attention in fundamental studies [16]. This study found that LINC00987 expression is enhanced in osteosarcoma cells and that inhibition of LINC00987 expression inhibited the proliferation, migration, and invasion of osteosarcoma cells. This study provides evidence that LINC00987 is an oncogene and therapeutic target. A previous study found that LINC00987 expression was increased in patients with acute myeloid leukemia and osteosarcoma and was closely related to poor prognosis $[9,17]$. These studies also support that LINC00987 is an oncogene, similar to our results.

IncRNAs act as competitive endogenous RNAs to sponge miRNAs and regulate their target mRNA expression transcriptionally or posttranscriptionally in osteosarcoma $[18,19]$. In this study, we found that LINC00987 acts as a sponge for miR-376a-5p. Previous study found that miR-376a-5p expression was reduced in lymphoma and miR-376a-5p knockdown enhanced lymphoma proliferation and apoptosis by targeting FOXP2 [20]. Additionally, miR-376a-5p reduced glioblastoma migration and invasion [21]. There result showed that miR-376a-5p is an antioncogene in 
A

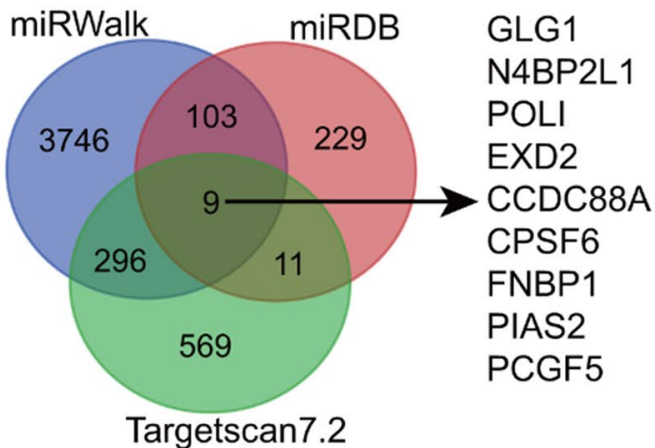

B

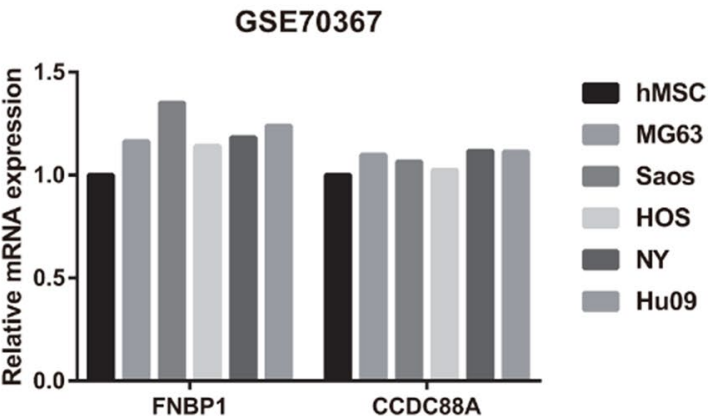

D

C

\section{MUT-3' UTR-FNBP1: 5'-AGUUCCUCUUGCUGUUUAUCUUAGAUA-3' WT-3' UTR-FNBP1: 5'-AGUUCCUCUUGCUGUUUAUGAAUCUAA-3' miR-376a-5p \\ | | | | | | | 3'-AUGAGUAUCUUCCUCUUAGAUG-5'}

E

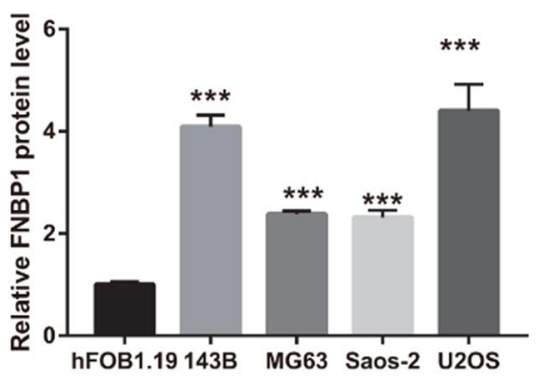

hFOB1.19 143B MG63 Saos-2 U2OS

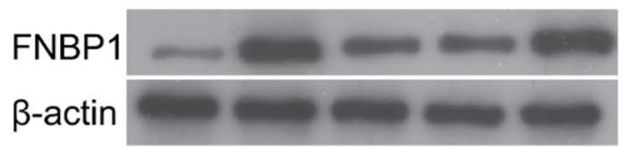

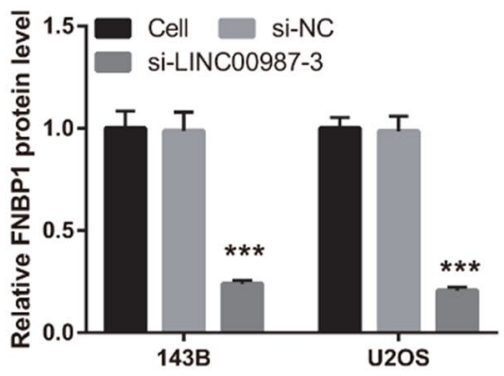

si-LINC Cell si-NC 00987-3 Cell si-NC 00987-3

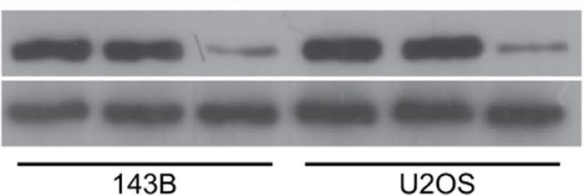

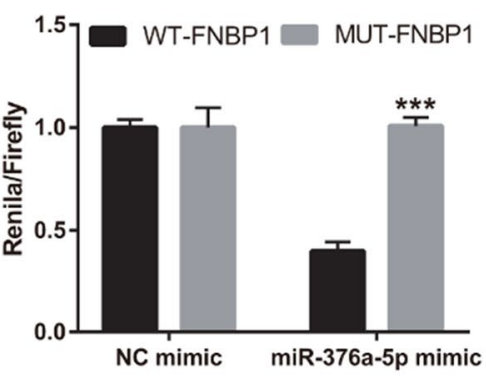

si-LINC00987-3+NC inhibitor $\bar{\Phi} 4^{4}$ si-LINC00987-3+miR inhibitor

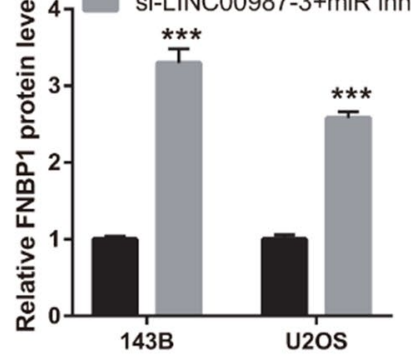

si-LINC00987-3
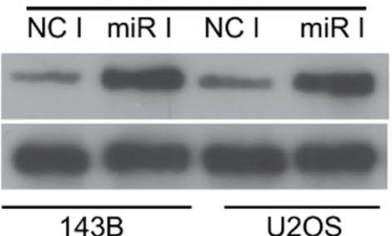

Fig. 6 FNBP1 is the target of miR-376a-5p. A The intersection between the potential mRNAs analyzed using Targetscan 7.2, miRDB, and miRWalk. B FNBP1 and CCDC88A expression in osteosarcoma cells and human mesenchymal stem cells (hMSCs) analyzed using the GEO database. C The wild-type and mutated binding sites between miR-376a-5p and FNBP1. D Luciferase reporter assay results. E mRNA expression and protein level of FNBP1 measured using qRT-PCR and western blotting, respectively, in the conditionally immortalized human fetal osteoblastic cell line hFOB1.19 and human osteosarcoma cell lines (143B, U2OS, MG63, and Saos-2), in 143B and U2OS cells transfected with three si-LINC00987 sequences and negative control siRNA (si-NC), and in 143B and U2OS cells co-transfected with si-LINC00987-3 and NC inhibitor (si-LINC00987-3 + NC inhibitor) or miR-376a-5p inhibitor (si-LINC00987-3 + miR inhibitor)

lymphoma and glioblastoma. However, effect of miR-376a-5p in osteosarcoma has not been elucidated. This study was the first to report that miR-376a-5p expression was reduced in osteosarcoma cells and that silencing miR-376a-5p promotes osteosarcoma development and attenuates the anticancer effect of LINC00987 inhibition in osteosarcoma. These results showed that miR-376a-5p is an antioncogene in osteosarcoma. Furthermore, it was found that miR376a-5p targets FNBP1 (also known as FNBP17), a member of the forming-binding protein family. FNBP1 expression was increased in pediatric medulloblastoma, which may act as prognostic markers and therapeutic targets [22]. Previous studies found that FNBP1 was overexpressed in invasive breast cancer cells and ductal carcinomas, regulating the cytoskeleton and metastasis [23, 24]. FNBP1 promoted invadopodia formation and invasive capacity in bladder 
Fig. 7 Mechanism diagram: LINC00987 reduces the binding of miR-376a-5p and 3'-UTR FNBP1 by sponging miR$376 a-5 p$, thereby increasing the level of FNBP1 protein and promoting the proliferation, migration and invasion of osteosarcoma

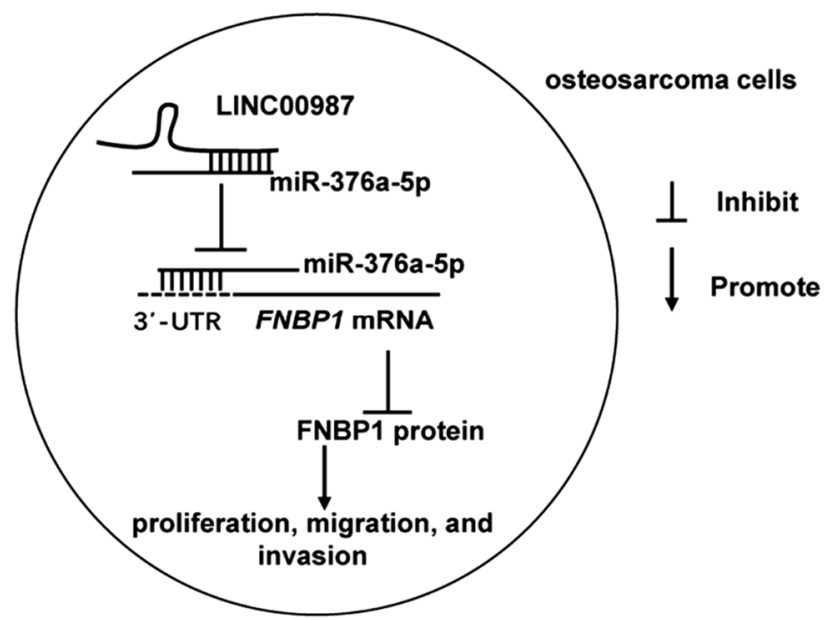

tumor cells [25]. These results showed that FNBP1 expression is increased in pediatric medulloblastoma, breast cancer cells and ductal carcinomas, and bladder tumor, which is an oncogene. Similarly, in this study, we found that FNBP1 is overexpressed in osteosarcoma. Additionally, silencing of LINC00987 inhibited FNBP1 levels, whereas silencing miR-376a-5p promoted FNBP1 levels, through posttranscriptional regulation in osteosarcoma. These results highlight that LINC00987 regulates the proliferation, migration, and invasion of osteosarcoma cells by adsorbing miR-376a-5p and posttranscriptionally regulating the expression of FNBP1.

\section{Conclusion}

Silencing LINC00987 inhibits proliferation, migration, and invasion of osteosarcoma cells by sponging miR-376a-5p to regulate FNBP1 expression. This study provides a potential therapeutic target for osteosarcoma.

Acknowledgements Not applicable.

Authors' contributions RC participated in the design of the study and writing draft manuscript. JS carried out qRT-PCR assay, western blotting, and statistical analysis. WZ, YL, and ZH carried out cell culture and cell functional experiment. ZL participated in the design of the study and the revision of draft manuscript. All authors read and approved the final manuscript.

Funding Not applicable.

Data availability All data generated or analysed during this study are included in this published article [and its Additional file].

Declarations

Ethics approval and consent to participate Not applicable.

Consent for publication Not applicable.

Competing interests The authors declare no competing interests.

Open Access This article is licensed under a Creative Commons Attribution 4.0 International License, which permits use, sharing, adaptation, distribution and reproduction in any medium or format, as long as you give appropriate credit to the original author(s) and the source, provide a link to the Creative Commons licence, and indicate if changes were made. The images or other third party material in this article are included in the article's Creative Commons licence, unless indicated otherwise in a credit line to the material. If material is not included in the article's Creative Commons licence and your intended use is not permitted by statutory regulation or exceeds the permitted use, you will need to obtain permission directly from the copyright holder. To view a copy of this licence, visit http://creativecommons.org/licenses/by/4.0/.

\section{References}

1. Geller DS, Gorlick R. Osteosarcoma: a review of diagnosis, management, and treatment strategies. Clin Adv Hematol Oncol. 2010;8:705-18. 
2. Da W, Tao Z, Meng Y, Wen K, Zhou S, Yang K, Tao L. A 10-year bibliometric analysis of osteosarcoma and cure from 2010 to 2019. BMC Cancer. 2021;21:115.

3. Ando K, Heymann MF, Stresing V, Mori K, Rédini F, Heymann D. Current therapeutic strategies and novel approaches in osteosarcoma. Cancers (Basel). 2013;5:591-616.

4. Fang Y, Fullwood MJ. Roles, Functions, and Mechanisms of Long Non-coding RNAs in Cancer. Genomics Proteomics Bioinform. $2016 ; 14: 42-54$.

5. Rafiee A, Riazi-Rad F, Havaskary M, Nuri F. Long noncoding RNAs: regulation, function and cancer. Biotechnol Genet Eng Rev. 2018;34:153-80.

6. Zhang W, Ren X, Qi L, Zhang C, Tu C, Li Z. The value of IncRNAs as prognostic biomarkers on clinical outcomes in osteosarcoma: a meta-analysis. BMC Cancer. 2021;21:202.

7. Tan J, Liang H, Yang B, Zhu S, Wu G, Li L, Liu Z, Li L, Qi W, Li S, Lin L. Identification and analysis of three hub prognostic genes related to osteosarcoma metastasis. J Oncol. 2021;2021:6646459.

8. Ghafouri-Fard S, Shirvani-Farsani Z, Hussen BM, Taheri M. The critical roles of IncRNAs in the development of osteosarcoma. Biomed Pharmacother. 2021;135:111217.

9. Sun K, Zhao J. A risk assessment model for the prognosis of osteosarcoma utilizing differentially expressed IncRNAs. Mol Med Rep. 2019;19:1128-38.

10. Cao R, Shao J, Hu Y, Wang L, Li Z, Sun G, Gao X. microRNA-338-3p inhibits proliferation, migration, invasion, and EMT in osteosarcoma cells by targeting activator of $90 \mathrm{kDa}$ heat shock protein ATPase homolog 1. Cancer Cell Int. 2018;18:49.

11. Livak KJ, Schmittgen TD. Analysis of relative gene expression data using real-time quantitative PCR and the 2(-Delta Delta C(T)) Method. Methods. 2001;25:402-8.

12. Paraskevopoulou MD, Vlachos IS, Karagkouni D, Georgakilas G, Kanellos I, Vergoulis T, Zagganas K, Tsanakas P, Floros E, Dalamagas T, Hatzigeorgiou AG. DIANA-LncBase v2: indexing microRNA targets on non-coding transcripts. Nucleic Acids Res. 2016;44:D231-238.

13. Agarwal V, Bell GW, Nam JW, Bartel DP. Predicting effective microRNA target sites in mammalian mRNAs. Elife. $2015 ; 4: e 05005$.

14. Chen $Y$, Wang X. miRDB: an online database for prediction of functional microRNA targets. Nucleic Acids Res. 2020;48:D127-d131.

15. Isakoff MS, Bielack SS, Meltzer P, Gorlick R. Osteosarcoma: Current treatment and a collaborative pathway to success. J Clin Oncol. 2015;33:3029-35.

16. Wang C, Jing J, Cheng L. Emerging roles of non-coding RNAs in the pathogenesis, diagnosis and prognosis of osteosarcoma. Invest New Drugs. 2018;36:1116-32.

17. Li G, Gao Y, Li K, Lin A, Jiang Z. Genomic analysis of biomarkers related to the prognosis of acute myeloid leukemia. Oncol Lett. 2020;20:1824-34.

18. Wang JY, Yang Y, Ma Y, Wang F, Xue A, Zhu J, Yang H, Chen Q, Chen M, Ye L, Wu H, Zhang Q. Potential regulatory role of IncRNA-miRNAmRNA axis in osteosarcoma. Biomed Pharmacother. 2020;121:109627.

19. Kushlinskii NE, Fridman MV, Braga EA. Long non-coding RNAs as competitive endogenous RNAs in osteosarcoma. Mol Biol (Mosk). 2020;54:776-801.

20. Yao S, Liu Y, Yao Z, Zhao Y, Wang H, Xu Y, Zhang J, Li J, Yang S. MicroRNA-376a regulates cell proliferation and apoptosis by targeting forkhead box protein P2 in lymphoma. Oncol Lett. 2018;16:3169-76.

21. Choudhury Y, Tay FC, Lam DH, Sandanaraj E, Tang C, Ang BT, Wang S. Attenuated adenosine-to-inosine editing of microRNA-376a* promotes invasiveness of glioblastoma cells. J Clin Invest. 2012;122:4059-76.

22. Huang P, Guo YD, Zhang HW. Identification of Hub genes in pediatric medulloblastoma by multiple-microarray analysis. J Mol Neurosci. 2020;70:522-31.

23. Suman P, Mishra S, Chander H. High formin binding protein 17 (FBP17) expression indicates poor differentiation and invasiveness of ductal carcinomas. Sci Rep. 2020;10:11543.

24. Suman P, Mishra S, Chander H. High expression of FBP17 in invasive breast cancer cells promotes invadopodia formation. Med Oncol. 2018;35:71.

25. Yamamoto H, Sutoh M, Hatakeyama S, Hashimoto Y, Yoneyama T, Koie T, Saitoh H, Yamaya K, Funyu T, Nakamura T, Ohyama C, Tsuboi S. Requirement for FBP17 in invadopodia formation by invasive bladder tumor cells. J Urol. 2011;185:1930-8.

Publisher's Note Springer Nature remains neutral with regard to jurisdictional claims in published maps and institutional affiliations. 